\title{
Modeling and Analysis of Thermoelectric Modules
}

\author{
Simon Lineykin and Shmuel Ben-Yaakov, Member, IEEE
}

\begin{abstract}
The objective of this paper is to develop a SPICEcompatible equivalent circuit of a thermoelectric module. A methodology is developed for extracting the parameters of the proposed model from manufacturers' data of thermoelectric coolers (TECs) and thermoelectric generators (TEGs). The model could be helpful in analyzing the drive requirements of TECs and loading effects of TEGs. The present model is compatible with PSPICE or other electric circuit simulators. An important feature of the model is its ability to generate small-signal transfer functions that can be used to design feedback networks for temperature control applications.
\end{abstract}

Index Terms-Active cooling, modeling, temperature control, thermoelectric generator (TEG), thermoelectricity.

\section{INTRODUCTION}

A THERMOELECTRIC module (TEM) is a solid-state energy converter. It normally consists of an array of $2 N$ pellets from dissimilar semiconductor material ( $\mathrm{p}$ and $\mathrm{n}$ type) that make up $N$ thermoelectric couples which are joined thermally in parallel and electrically in series, as shown in Fig. 1. The TEM can be used for cooling, heating, and energy generation [1], [2]. As a thermoelectric cooler (TEC), the TEM has already found applications in thermal management and control of microelectronic devices such as diode lasers and CPUs. As a thermoelectric generator (TEG), the TEM could be used to produce electric power in remote locations when temperature gradients are available [1], [2].

The objective of this paper is to develop a SPICE compatible equivalent circuit of a TEM. An equivalent circuit is a convenient tool for electronic engineers. It helps to present the problem in electronic circuit terms, helps to understand its functionality, and facilitates the solving of cooling or powergeneration problems without the need for expertise in thermal engineering.

In [3], the authors proposed an equivalent circuit topology for a TEC. In the present study, the analysis given by the study in [3] is extended to include both the TEC and TEG operation by a universal equivalent circuit. Another contribution of this work, which may have great practical importance, is a proposed

Paper IPCSD-06-094, presented at the 2005 IEEE Applied Power Electronics Conference and Exposition, Austin, TX, March 6-10, and approved for publication in the IEEE TRANSACTIONS ON INDUSTRY APPLICATIONS by the Power Electronics Devices and Components Committee of the IEEE Industry Applications Society. Manuscript submitted for review December 7, 2005 and released for publication November 6, 2006

The authors are with the Power Electronics Laboratory, Department of Electrical and Computer Engineering, Ben-Gurion University of the Negev, Beer-Sheva 84105, Israel (e-mail: simonl@ee.bgu.ac.il; sby@ee.bgu.ac.il).

Color versions of one or more of the figures in this paper are available online at http://ieeexplore.ieee.org.

Digital Object Identifier 10.1109/TIA.2006.889813

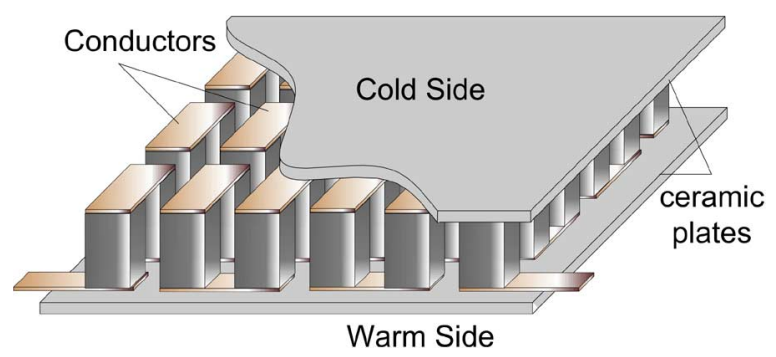

Fig. 1. Typical one-stage 18-couples TEM with two electrodes and ceramic plates.

method for extracting the model parameters from the specifications found in commercial datasheets—as detailed below.

\section{PRINCIPLES OF OPERATION}

Five main physical processes take place in a TEM [1]: Thermal convection-the phenomenon referred to as the Fourier process, described by a physical constant $k$ (in watt per meter Kelvin), which is determined by the thermal conductivity and geometry of the pellet.

The equation of heat transfer in a pellet is

$$
q=-k A \frac{\partial T}{\partial x} \approx-k \frac{A}{L} \Delta T=-\frac{\Delta T}{2 \Theta}
$$

where $x$ is the distance along the heat flow path, $L / A$ is a geometry factor, $L$ is the height of the pellet (in meter), $A$ is the cross-sectional area of the pellet (in square meter), $T$ is the temperature (in Kelvin), and $q$ is the heat (in watt). $\Theta$ (in Kelvin per watt) is the thermal resistance of the couple of pellets and is expressed as

$$
\Theta=\frac{1}{k} \frac{L}{2 A}
$$

The total thermal resistance of the $N$ couples of a module, which are connected thermally in parallel, will be

$$
\Theta_{m}=\Theta / N \text {. }
$$

Joule heating is the physical process of heat dissipation in the resistive elements. The electrical resistance $R$ of a couple of pellets is

$$
\begin{aligned}
R & =\rho \frac{2 L}{A} \\
q_{j} & =I^{2} R_{m}
\end{aligned}
$$




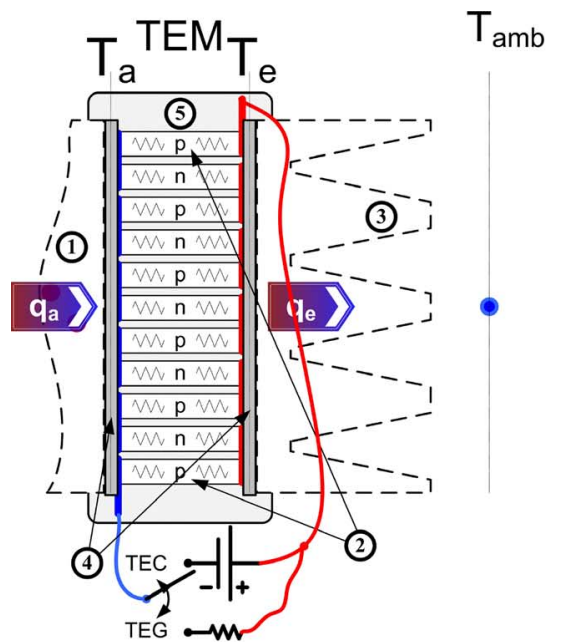

(a)

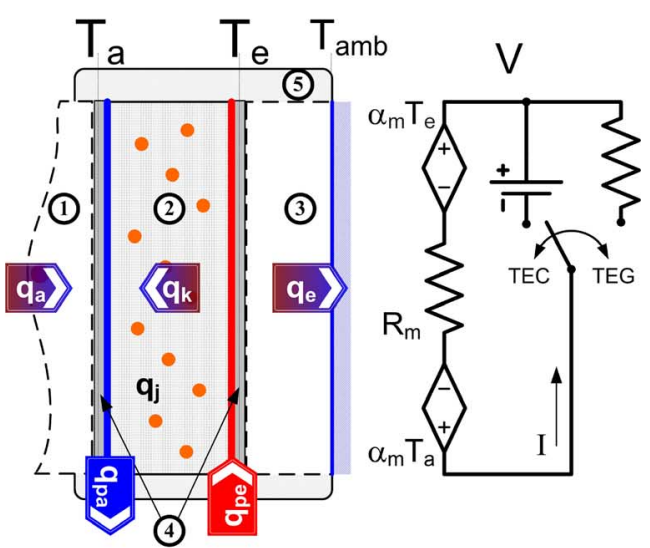

(b)

Fig. 2. Energy equilibrium in a TEM connected to a thermal load and heat sink. (a) Schematic view of the system. (b) One-dimensional representation of the problem including the electrical port. 1: thermal load; 2: TEM; 3: heat sink; 4: ceramic plates; 5: thermal insulation.

where $\rho$ is the resistivity of the material (in ohm meter), $q_{j}$ is the Joule heating of TEM (in watt), $I$ is the electric current (in ampere), and $R_{m}$ is the total resistance of the TEM with $N$ couples

$$
R_{m}=R \cdot N
$$

The Seebeck power generation is a process by which heating (or cooling) of the junction of two dissimilar materials generates an electrical potential across the junction

$$
\pi=\alpha T
$$

where $\pi$, referred to as Peltier coefficient, is the electrical potential (in volt) of the junction and $\alpha$ (in volt per Kelvin) is the Seebeck coefficient corresponding to a specific pair of materials. For $N$ couples in a TEM

$$
\alpha_{m}=\alpha N
$$

The electromotive force (EMF) of the TEM will, thus, be

$$
U=\alpha_{m}\left(T_{a}-T_{e}\right) .
$$

The heat-absorbing junctions are defined as the $(a)$ side, whereas the heat-emitting junctions are defined as the $(e)$ side of the TEM. The $T_{a / e}$ is the temperature of the corresponding junction.

Peltier cooling/heating is the phenomenon of the absorption (or dissipation) of heat by a junction between two dissimilar materials when electrical current flows through the junction. The heat $q_{p a / p e}$ absorbed/emitted by the TEM is

$$
q_{p a / p e}=\alpha_{m} T_{a / e} I .
$$

The Peltier cooling/heating $q_{p a / p e}$ is sensitive to current direction; thus, half of the TEM's junctions that conduct the current in one direction will absorb heat, whereas the other half, where current flows in opposite direction, will release heat.
An additional thermoelectric phenomenon-the Thompson phenomenon-is described by the Thompson coefficient $\tau=$ $d \alpha / d T$ (in volt per square Kelvin). The effect of this phenomenon is small [1], [2] and is therefore neglected in this paper.

Fig. 2(a) is a schematic representation of a system comprising a TEM, thermal load, and heat exchanger. When the system operates in the TEC mode, an electrical power supply is connected to the electric port, and heat is pumped from the thermal load side $(a)$ where heat is absorbed to the heat sink side $(e)$ where heat is released. In the case of power generator operation mode, the electric load is connected to the electric port, while the system absorbs thermal energy from the temperature difference between the $(a)$ and $(e)$ sides.

\section{SteAdy-State ANALYSis}

Fig. 2(b) is a schematic representation of the TEM, together with a thermal load and heat sink shown as a 1-D thermal system. The incoming heat generated by the thermal load is defined as $q_{a}$; Peltier cooling/heating $q_{p a / p e}$ occurs at the junctions and is assumed to be concentrated at the interfaces. The Joule heating $q$ is assumed to be uniformly generated throughout the volume of the TEM, and $q_{e}$ is the heat removed from the system via the heat sink. The 1-D steady-state heat conduction equation for solid with energy generation is

$$
\frac{\partial^{2}}{\partial x^{2}} T+\frac{\dot{q}}{k}=0
$$

where $\dot{q}=q_{j} /(A L)$ is the heat generated per unit volume. By applying the notation of (2), one can rewrite (11) for the temperature boundary conditions at $x=0$ and $x=L$.

$$
\begin{aligned}
\frac{\partial^{2}}{\partial x^{2}} T+\frac{q_{j}}{A L} \cdot \frac{\Theta_{m} A}{L} & =0 \\
T \rightarrow T_{a} @ x & =0 \\
T \rightarrow T_{e} @ x & =L .
\end{aligned}
$$


TABLE I

THERMAl to Electrical ANALOgy

\begin{tabular}{|l|l|l|l|}
\hline Thermal Quantities & Units & ANalogous Electrical QUantities & Units \\
\hline Heat, q & $\mathrm{W}$ & Current, I & $\mathrm{A}$ \\
\hline Temperature, $\mathrm{T}$ & $\mathrm{K}$ & Voltage, $\mathrm{V}$ & $\mathrm{V}$ \\
\hline Thermal Resistance, $\Theta$ & $\mathrm{K} \mathrm{W}^{-1}$ & Resistance, $\mathrm{R}$ & $\Omega$ \\
\hline Heat capacity, C & $\mathrm{J} \mathrm{K}^{-1}$ & Capacitance, $\mathrm{C}$ & $\mathrm{F}$ \\
\hline Absolute zero temperature & $0 \mathrm{~K}$ & Ground & $0 \mathrm{~V}$ \\
\hline
\end{tabular}

By solving (12) and substituting the result into (1), one gets the expression for the heat flows through each side of the solid with thermal generation

$$
\begin{aligned}
q(x \rightarrow 0) & =-\frac{T_{e}-T_{a}}{\Theta_{m}}-\frac{I^{2} R_{m}}{2} \\
q(x \rightarrow L) & =-\frac{T_{e}-T_{a}}{\Theta_{m}}+\frac{I^{2} R_{m}}{2} .
\end{aligned}
$$

Hence, irrespective of the value of the temperature gradient, the Joule heat can be considered as equally divided between the (a) and (b) sides of the TEM.

By applying the energy balance concept for steady-state, taking into account the Peltier heating/cooling positive/negative heat sources at the interfaces [see Fig. 2(b)], and applying the result of (13), one can express the energy equilibrium at both sides of the TEM. For the absorbing side $(a)$, one can write

$$
q_{a}-\alpha_{m} T_{a} I=-\frac{T_{e}-T_{a}}{\Theta_{m}}-\frac{I^{2} R_{m}}{2}
$$

and for the emitting side $(e)$

$$
q_{e}-\alpha_{m} T_{e} I=-\frac{T_{e}-T_{a}}{\Theta_{m}}+\frac{I^{2} R_{m}}{2}
$$

where $q_{a}$ represents heat absorption at the $(a)$ side, $q_{e}$ is the heat emission at the $(e)$ side, and $T_{a}$ and $T_{e}$ are the temperatures of (a) and (e) sides in Kelvin.

The voltage $V$ of the electrical port of the module is a function of the current and resistance (6), and the EMF (9)

$$
V=I R_{m}+\alpha_{m} \Delta T
$$

where $\Delta T=T_{e}-T_{a}$ [Fig. 2(b)].

One approach for solving heat transfer problems is to apply an equivalent electrical circuit scheme [4]. This approach was adopted in this paper to describe the TEM system with direct energy conversion. All nonelectrical processes are described in terms of electrical analogies, and dependent sources represent their interconnections. Table I shows the physical parameters of the thermal system and corresponding parameters of the equivalent electric circuit. By applying (14)-(16) for the $(a)$

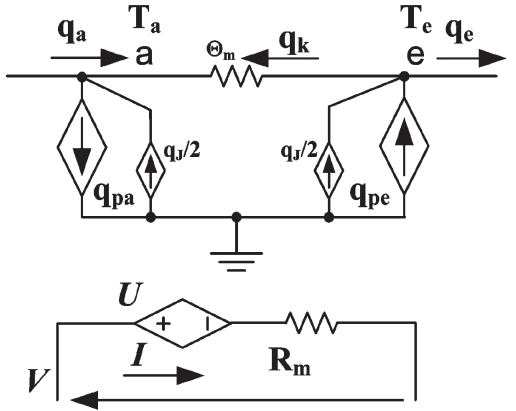

Fig. 3. Equivalent circuit of a TEM under a steady-state condition.

and $(e)$ junctions, an electrical equivalent circuit of the TEM (Fig. 3) can be constructed.

\section{Calculation of the Parameters of the Model From THE MANUFACTURERS' DATASHEETS}

As described by the study in [5], the construction of the TEC and the TEG is about the same. Consequently, the same equivalent circuit model can be used in simulating their behavior. However, manufacturers use different ways to specify the data for TECs and TEGs. In the following, we propose a method for extracting the fundamental TEC and TEG parameters directly from the datasheets.

Manufacturers of TECs ${ }^{1-3}$ use the following parameters to specify their product: $\Delta T_{\max }$ is the largest temperature differential (in Kelvin) that can be obtained between the "cold" $(a)$ and the "hot" $(e)$ sides of a thermally insulated TEC for the given value of "hot"-side temperature $T_{h} . I_{\max }$ and $U_{\max }$ are the input dc current (in ampere) and voltage (in volt) which will produce the $\Delta T_{\max }$ across a TEC.

By applying (14)-(16), one can express the datasheets' parameters by setting $T_{e}$ to $T_{h}, T_{a}$ to $T_{h}-\Delta T$, and $q_{a}$ to zero (thermal insulation), and eliminating $V$. The expression for $\Delta T$ is found to be

$$
\Delta T=\frac{I \cdot \Theta_{m}\left(2 \alpha_{m} T_{h}-I \cdot R_{m}\right)}{2\left(1+I \cdot \Theta_{m} \cdot \alpha_{m}\right)}
$$

\footnotetext{
${ }^{1}$ Kryotherm Company products. [Online]. Available: http://www kryotherm.ru

${ }^{2}$ Beijing Huimao Cooling Equipment Company products. [Online]. Available: http://www.huimao.com

${ }^{3}$ Marlow Industries products. [Online]. Available: http://www.marlow.com
} 
By taking the derivative of $\Delta T$ with respect to $I$ and setting it to zero, one can derive the value of the current that will produce $\Delta T_{\max }$

$$
I_{\max }=\frac{\sqrt{1+2 T_{h} Z}-1}{\alpha_{m} \Theta_{m}}
$$

where $Z$ is the figure of merit of the TEM

$$
Z=\frac{\alpha^{2} \Theta}{R}=\frac{\alpha_{m}^{2} \Theta_{m}}{R_{m}} .
$$

Substitution of (18) into (17) gives the expression for $\Delta T_{\max }$

$$
\Delta T_{\max }=T_{h}+\frac{1-\sqrt{1+2 T_{h} Z}}{Z} .
$$

Now, setting $T_{e}$ as $T_{h}, T_{a}$ as $T_{h}-\Delta T$, and $q_{a}$ equal to zero, in the set of (14)-(16), and eliminating $\Delta T$, one gets the expression for $V$

$$
V=\frac{2 I R_{m}+\alpha_{m} \Theta_{m}\left(I R_{m}+2 \alpha_{m} T_{h}\right)}{2+2 I \alpha_{m} \Theta_{m}}
$$

The substitution of (18) into (21) gives, after some simplification,

$$
U_{\max }=\left.V\right|_{I \rightarrow I_{\max }}=\alpha_{m} T_{h}
$$

By applying (18), (20), and (22), one can use the set of data: $T_{h}, \Delta T_{\max }, U_{\max }$, and $I_{\max }$ for calculating the fundamental parameters of the proposed model

$$
\begin{aligned}
\alpha_{m} & =\frac{U_{\max }}{T_{h}} \\
R_{m} & =\frac{U_{\max }}{I_{\max }} \frac{\left(T_{h}-\Delta T_{\max }\right)}{T_{h}} \\
\Theta_{m} & =\frac{\Delta T_{\max }}{I_{\max } U_{\max }} \frac{2 T_{h}}{\left(T_{h}-\Delta T_{\max }\right)} .
\end{aligned}
$$

The regular set of data in the datasheet of TEGs (for example, Hi-Z technology) ${ }^{4}$ includes the thermal conditions for which the parameters are specified: the temperature of the "hot" side $T_{a}$, the temperature of the "cold" side $T_{e}$, power at the matched load $W_{m}$ (load is matched to internal resistance), load voltage at the matched load $U_{m}$, and maximum efficiency $\eta_{\text {opt }}$. Some manufacturers give $\eta_{m}$-efficiency for the matched load.

By using this data, one can easily calculate the electrical parameters of the equivalent circuit directly from the datasheets

$$
\begin{aligned}
R_{m} & =\frac{U_{m}^{2}}{W_{m}} \\
\alpha_{m} & =\frac{2 U_{m}}{T_{a}-T_{e}} .
\end{aligned}
$$

Difficulties emerge when calculating thermal resistance. For this purpose, one needs to take into account the efficiency. A method for finding the efficiency as a function of the load is given by the study in [1].

If the load resistance $R_{\text {load }}$ is defined as $R_{\text {load }}=m \cdot R_{m}$, the current can be expressed as

$$
I=\alpha_{m} \frac{T_{a}-T_{e}}{R_{m}(1+m)} .
$$

The efficiency [from (14)-(16), and (28)] is found as

$$
\eta(m)=\frac{I^{2} R_{m}}{q_{a}}=\frac{2 \cdot Z \cdot m \cdot\left(T_{a}-T_{e}\right)}{2(1+m)^{2}+Z\left(T_{a}+2 m T_{a}+T_{e}\right)} .
$$

For the matched load $\eta(m=1)$, the efficiency is found to be

$$
\eta_{m}=\eta(m=1)=\frac{1}{2} \cdot \frac{T_{a}-T_{e}}{T_{a}-\frac{1}{4}\left(T_{a}-T_{e}\right)+\frac{2}{Z}} .
$$

By solving (30) for $Z$, one gets

$$
Z=\frac{8 \eta_{m}}{T_{a}\left(2-3 \eta_{m}\right)-T_{e}\left(2+\eta_{m}\right)} .
$$

In the case of a manufacturer providing the maximum possible efficiency, denoted here as $\eta_{\text {opt }}$, the relation $m$ in (29) between the load and internal resistances is found by the study in [1] as

$$
m_{\mathrm{opt}}=\sqrt{1+\frac{T_{a}+T_{e}}{2} Z}
$$

By substituting this optimum value of $m$, denoted here as $m_{\mathrm{opt}}$, into the expression for efficiency (29), the expression for the maximum possible efficiency $\left(\eta_{\text {opt }}\right)$ is found to be

$$
\eta_{\mathrm{opt}}=\eta\left(m_{\mathrm{opt}}\right)=\eta_{c} \frac{m_{\mathrm{opt}}-1}{m_{\mathrm{opt}}+\frac{T_{e}}{T_{a}}}
$$

where

$$
\eta_{c}=\frac{T_{a}-T_{e}}{T_{a}}
$$

By solving (33) for $m_{\mathrm{opt}}$ and then (32) for $Z$, one finds that, in the case of a given $\eta_{\mathrm{opt}}, Z$ can be calculated as

$$
Z=\frac{2}{\left(T_{a}+T_{e}\right)}\left(\left(\frac{\eta_{c} T_{a}+\eta_{\mathrm{opt}} T_{e}}{\eta_{c} T_{a}-\eta_{\mathrm{opt}} T_{a}}\right)^{2}-1\right) .
$$

Finally, from (19), (26), and (27), the value of the thermal resistance of the TEG can be calculated

$$
\Theta_{m}=\frac{R_{m}}{\alpha_{m}^{2}} Z
$$




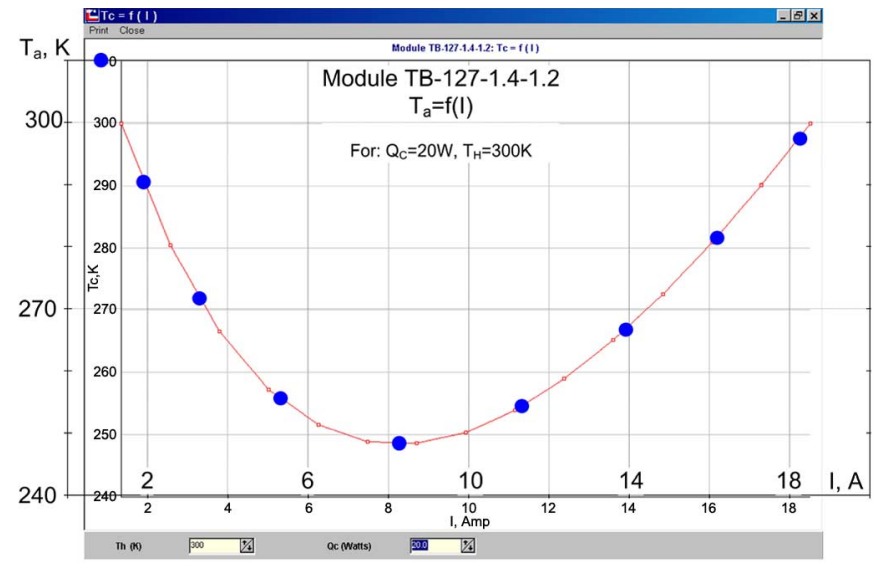

Fig. 4. Performance of TEM TB-127-1.4-1.2. Dotted line: simulation result obtained by the proposed model. Solid line: performance plot published by the manufacturer.

\section{EXAMPLES}

In this section, several examples of model applications are presented.

1) The TB-127-1.4-1.2 is one of the thermoelectric cooling modules available from Kryotherm. From the manufacturer's datasheets, $\Delta T_{\max }=70 \mathrm{~K}, I_{\max }=7.6 \mathrm{~A}$, and $U_{\text {max }}=15.9 \mathrm{~V}$, under the condition that $T_{h}=300 \mathrm{~K}$.

By applying (23)-(25), one can calculate the model parameters: $\alpha_{m}=0.053 \mathrm{~V} \cdot \mathrm{K}^{-1}, R_{m}=1.6 \Omega$, and $\Theta_{m}=1.5 \mathrm{~K} \cdot \mathrm{W}^{-1}$.

Fig. 4 shows the result of the application of a DC-Sweep simulation that reconstructs the performance plot of the TEM TB-127-1.4-1.2. The dotted line is the simulation result. The original performance plot was copied from the software, which was placed on the Internet by Kryotherm.

2) The TEG HZ-20 is manufactured by Hi-Z Technology. The data from the datasheets: for a hot-side temperature $T_{h}=230{ }^{\circ} \mathrm{C}$ and for a cold-side temperature $T_{c}=$ $30{ }^{\circ} \mathrm{C}, W_{m}=19 \mathrm{~W}, U_{m}=2.38 \mathrm{~V}, \eta_{\text {opt }}=4.5 \%$. From (26), (27), and (36), $\alpha_{m}=0.0238 \mathrm{~V} \cdot \mathrm{K}^{-1}, \Theta_{m}=0.589 \mathrm{~K}$. $\mathrm{W}^{-1}$, and $R_{m}=0.298 \Omega$.

Fig. 5 shows the DC-Sweep simulation of the HZ-20 TEG. The simulated variables are voltage $V$, current $I$, power $W$, and efficiency $\eta$, with the load as the sweep parameter. The coordinate system is voltage, power, and efficiency versus current. As in the previous case, the plot of simulation was superimposed on the one downloaded from the site of the manufacturer (shown by the solid lines). The dotted lines show the results of the module simulation dc-sweep, with the load as parameter.

\section{DynAmic BehaVior of A THERMAL TEM SyStem}

The direct energy conversion processes taking place in a TEM can be considered instantaneous and therefore do not influence the dynamics of the thermal system. That is, the dynamics is controlled by the thermal resistances and thermal

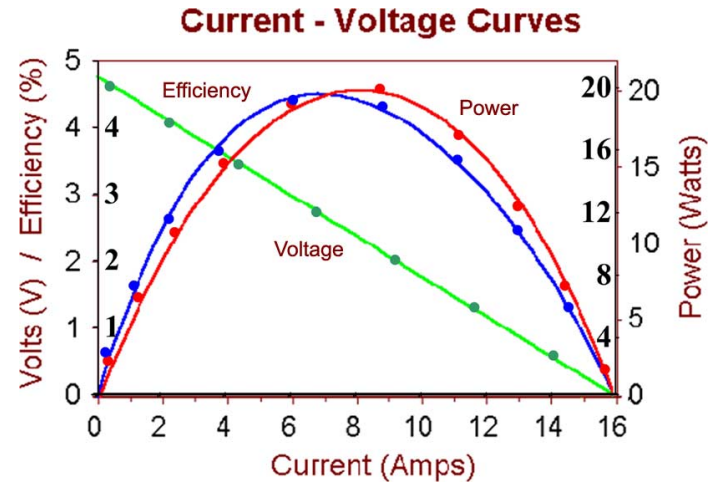

Fig. 5. DC-sweep simulation of the TEG HZ-20 performance. Dotted line: results of the computer simulation. Solid line: performance plot published by the manufacturer.

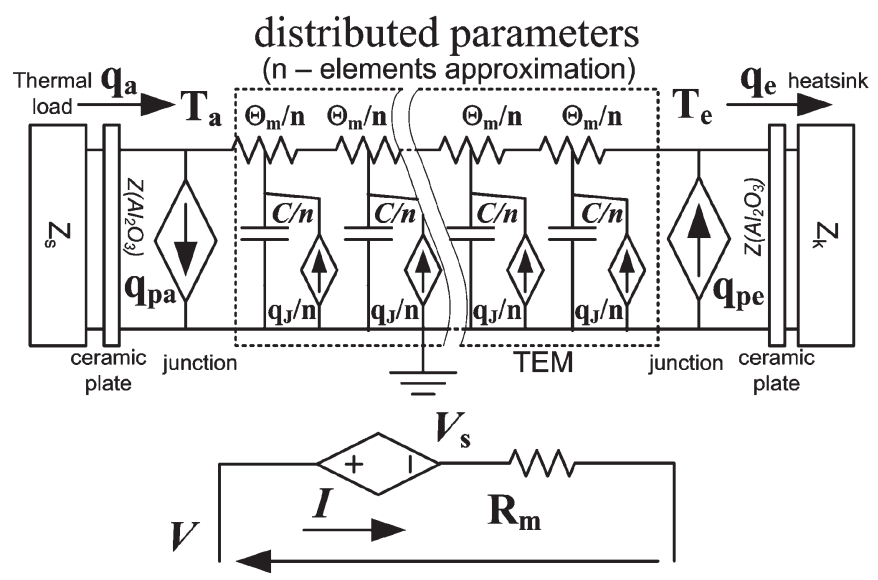

Fig. 6. Proposed equivalent circuit of a TEM, thermal load, and heat sink suitable for the numerical solution of the heat transform equation for the general case. $n$ is the number of nodes of the grid.

masses of the system [7]. The dynamic behavior of the system is described by a nonhomogenous differential heat transfer equation for solid [4]. The system under consideration is complex (see Fig. 2); it includes a solid with internal power generation (TEM), several sources of heat, and time-variable boundary conditions. There is no closed analytical solution for this kind of equations. Nevertheless, several options are available for solving the problem numerically. A commonly used approach would be to divide the solid body into a grid. Each spatial discretization can then be transformed into an equivalent thermal circuit in terms of electrical analogies (see Table I), as shown in Fig. 6. Clearly, the ceramic plates, heat sink, thermal load, etc., also need to be represented as lumped capacitances or thermal impedances using the suggested method or other common methods as proposed in [7]. By increasing the number of nodes in the model, one improves the precision of the solution. In [8], the authors use such a finite element model with a 42-element grid in describing a 1-D temperature distribution within a TEM. One can significantly decrease the number of grid nodes when the time constants of thermal load and heat sink are large. In this case, the stationary TEM behavior is sufficient in describing the transient cases investigated, so that there is no need for a transient TEM model. In such cases, the simplified model of the TEM, shown in Fig. 7, demonstrated a good agreement with 


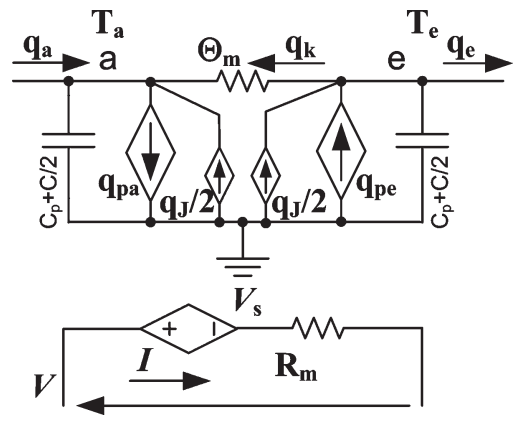

Fig. 7. Simplified model of a TEM in which the thermal resistance $\left(\Theta_{m}\right)$ and thermal capacitance $(C)$ of the TEM, as well as the thermal capacitance of attached ceramic plates $\left(C_{p}\right)$, are represented as lumped elements.

the experimental results. In cases where the thermal masses of the external elements are comparable or smaller than that of the TEM or when the high-frequency behavior needs to be investigated, one will have to increase the number of nodes in the model.

\section{COMPARISON OF THE EXPERIMENTAL DATA With MODEL Simulation}

The proposed model was verified by comparing computer simulations, that were carried out with the proposed model, to laboratory measurements that were conducted with a physical TEM. The experiments were carried out with a commercial TEM TB-127-1.4-1.2 (Kryotherm) having the dimensions of $40 \times 40 \times 3.6 \mathrm{~mm}$ and the performance characteristics (as cited by the manufacturer): $\Delta T_{\max }=70 \mathrm{~K}, I_{\max }=7.6 \mathrm{~A}$, and $U_{\max }=15.9 \mathrm{~V}$, under the conditions that $T_{h}=300 \mathrm{~K}$. This TEC module has 127 couples, the thickness of each of the ceramic plates is $1 \mathrm{~mm}$, and the length of each pellet is $1.4 \mathrm{~mm}$. By applying (23)-(25), the parameters of the model were calculated to be: $R_{m}=1.602 \Omega, \Theta_{m}=1.499 \mathrm{~K} \cdot \mathrm{W}^{-1}$, and $\alpha_{m}=0.0532 \mathrm{~V} \cdot \mathrm{K}^{-1}$. The lumped heat capacitance of the TEM was estimated to be $C=0.35 \mathrm{~J} \cdot \mathrm{K}^{-1}$, and the lumped heat capacitance of each one of the ceramic plates was calculated to be $\mathrm{Cp}=5.33 \mathrm{~J} \cdot \mathrm{K}^{-1}$. Data for the thermal volumetric heat capacity were taken from the study in [9] and the volumes of the ceramic plates and pellets from the datasheets. Thus, $C_{t}=\mathrm{Cp}+C / 2=5.68 \mathrm{~J} \cdot \mathrm{K}^{-1}$ (Fig. 7).

Two aluminum plates $(40 \times 40 \times 5 \mathrm{~mm})$ with thermocouples inserted into them were used for temperature measurements. The thermal resistance of the plates is assumed to be negligibly small, and their lumped heat capacity was calculated by

$$
C_{\mathrm{al}}=c \rho v
$$

where $c$ is the specific heat of aluminum $\left(0.896 \mathrm{~kJ} \cdot \mathrm{kg}^{-1}\right.$. $\left.\mathrm{K}^{-1}\right), \rho$ is the density of aluminum $\left(2707 \mathrm{~kg} \cdot \mathrm{m}^{-3}\right)$, and $v$ is the volume $\left(8 \times 10^{-6} \mathrm{~m}^{3}\right)$. In this specific case, the $C_{\mathrm{al}}$ is about $19 \mathrm{~J} \cdot \mathrm{K}^{-1}$. The experiments were conducted under thermally insulated condition. However, even though the system was intended to be thermally insulated, a small amount of heat leakage still existed. The value of thermal resistance of the
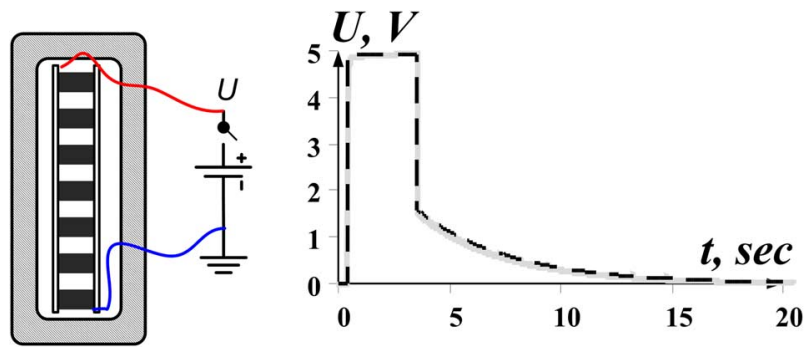

Fig. 8. Experimental setup and results of the TEM's time response. Gray thick line: result of the experiment on the physical module. Black dashed line: result of SPICE simulation using the equivalent circuit scheme of Fig. 6 or 7 with open circuited thermal ports

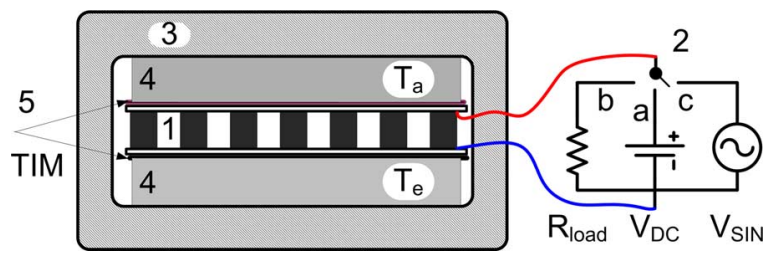

Fig. 9. Experimental setup. 1: TEM; 2: electrical port connected to a) dc source, b) load, and c) sine wave source; 3: thermal insulation; 4: aluminum plates with attached thermocouples; and 5: thermal interface material.

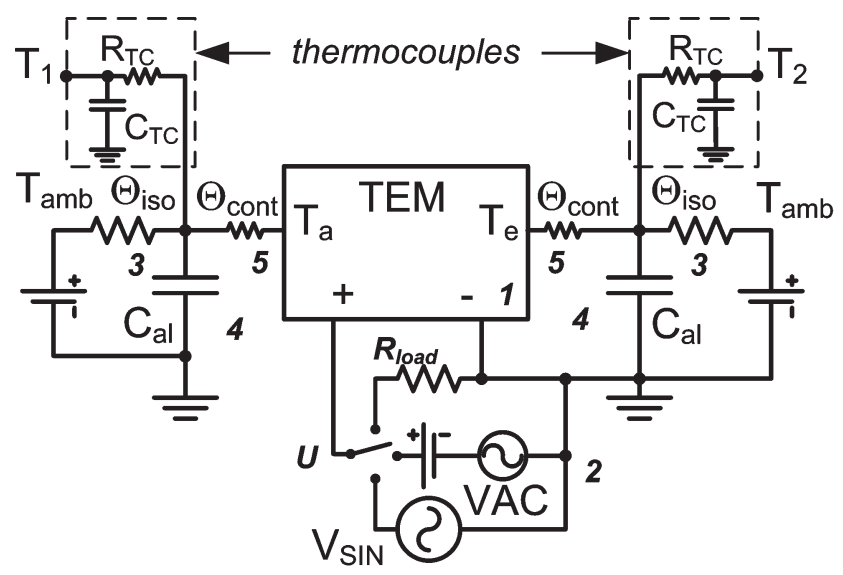

Fig. 10. PSPICE model for simulating the experimental system by the equivalent circuit of Fig. 6 or $7 . \Theta_{\text {iso }}$ is the thermal resistance of the thermal insulation. $C_{\mathrm{al}}$ is the thermal capacity of the aluminum plates. $\Theta_{\text {cont }}$ is the thermal resistance of the thermal contact between the TEM and the aluminum plates.

isolation was calculated from the steady-state measurement when applying a low input power

$$
\Theta_{\mathrm{iso}}=\frac{T_{\mathrm{av}}-T_{\mathrm{amb}}}{2 P_{e}} \approx 51.6\left(\mathrm{~K} \cdot \mathrm{W}^{-1}\right)
$$

where $T_{\mathrm{av}}=\left(T_{a}+T_{e}\right) / 2$, and $P_{e}=V \cdot I$. The thermal capacitance of isolation was neglected because of its large time constant (about $1 \mathrm{~h}$ in our case), whereas the experiments lasted for few seconds.

In addition, the thermal resistance of the thermally conducting grease between the TEM and the aluminum plates $\Theta_{\text {cont }}$ was taken into account. Its value was assumed to be $0.45 \mathrm{~K} \cdot \mathrm{W}^{-1}$ for the area of $16 \mathrm{~cm}^{2}$, which would be typical for the thermally conducting grease. The simulations were run 


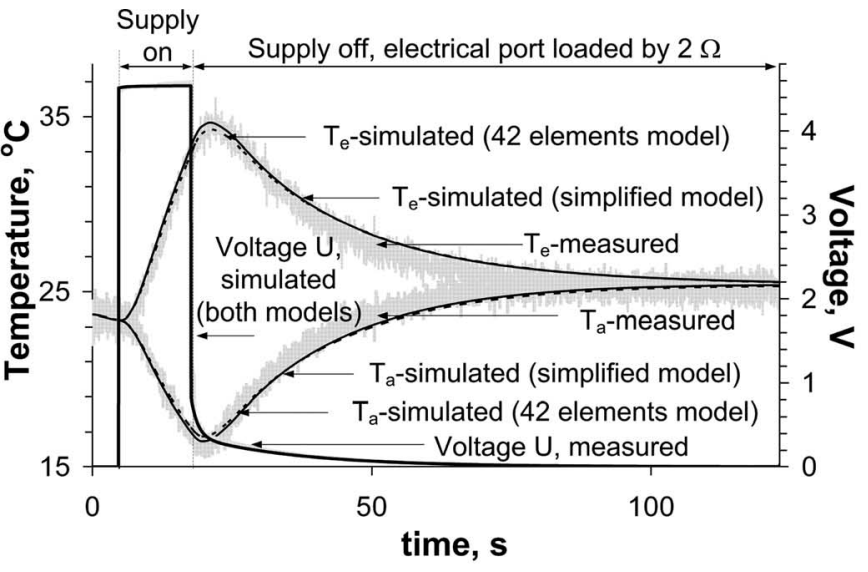

(a)

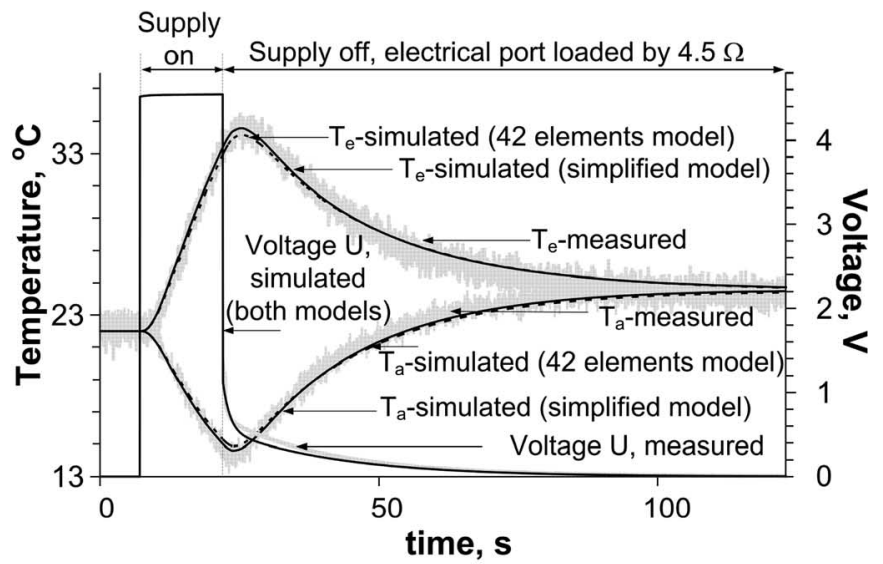

(b)

Fig. 11. Behavior of the TEM under a sequence of powering and loading at the electric port. (a) Electrical load: $2 \Omega$. (b) Electrical load: $4.5 \Omega$.

on the simplified model (Fig. 7) as well as on the 42-elements model (analogously to the one described by the study in [8]) shown in Fig. 6.

\section{A. Thermally Insulated TEM}

In this experiment, the module was thermally insulated (see Fig. 8). In the first phase of the experiment, a constant voltage was applied for several seconds to the electrical port. As a result, a temperature difference between the absorbing and emitting sides of the TEC was established. Then, the timedomain relaxation of the temperature difference to zero was observed by measuring the voltage of the open electrical port. The goal of the experiment was to check the dynamic characteristics of the model. The plot in Fig. 8 shows the good agreement between the simulation and experimental results.

\section{B. TEM With Thermal and Electrical Load}

The module was thermally loaded-inserted between two aluminum plates with thermocouples attached for temperature measurements (see Fig. 9). Both plates were insulated thermally from the ambient air. As in the previous experiment, the TEM was first "charged" with a dc voltage to specific temperature difference between its sides; then, the supply voltage was turned off. From that point, TEM was continuing its operation as a generator. Its output voltage $U$ and the temperatures of the aluminum plates were measured for different electrical loads. The equivalent circuit of the experimental setup is depicted in Fig. 10. It includes additional networks $\left(R_{\mathrm{TC}}\right.$ and $\left.C_{\mathrm{TC}}\right)$ to take into account the delays of the thermocouples that were used to measure the aluminum plates' temperatures $\left(T_{a}\right.$ and $\left.T_{e}\right)$. This extra time constant was estimated to be $1.6 \mathrm{~s}$.

Fig. 11 shows the experimental results together with the computer simulation results. The good agreement clearly shows that the model is valid not only for steady-state conditions (dc) but also for simulating the dynamic behavior under the experimental conditions. Due to the relative large time constants of the TEM's loads, the 42-element model of Fig. 6 and the simplified model of Fig. 7 show practically identical results.
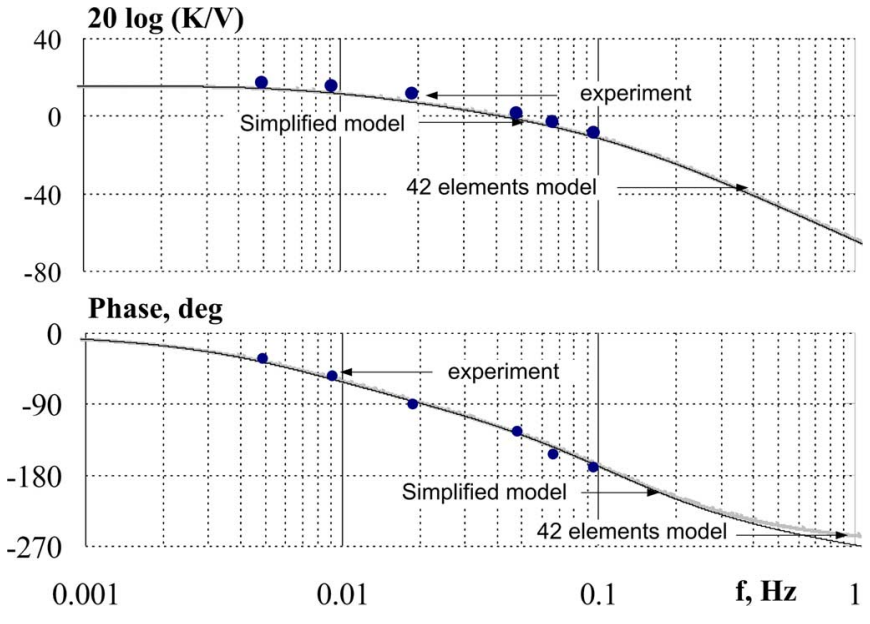

Fig. 12. Transfer function of the experimental system of Fig. 9. $X$ axis: Input voltage. $Y$ axis: Temperature difference between the aluminum plates on both sides of the TEC. Gray and black solid lines: Small-signal (ac) simulation result for 42-element TEM model (Fig. 6) and simplified model (Fig. 7). Points: Experimental results.

\section{Small-Signal Transfer Function of the TEM}

A prerequisite for a good controller design is knowledge of the system's frequency response (transfer function). An analytical method for calculating the poles and zeros of the transfer function of the TEC-based system is given by the study in [10]. However, since the TEC is a nonlinear system, the transfer function will be different for each operating (bias) point. The analytical derivation of the transfer function for all conditions of operation is thus a cumbersome process. The proposed model provides a simple way to get the transfer functions of the system directly from the large-signal model by just carrying out a small-signal (ac) simulation of the cooling system by an electronic circuit simulator such as PSPICE. An important feature of modern circuit simulators is the built-in linearization algorithm, about any operational point, that is run automatically as the first step of a small-signal (AC-Sweep) analysis. This ability, which is transparent to the user, alleviates the need for deriving a separate small-signal model for each studied system. 
The setup is the same as in Fig. 9, but in this case, the source generates a sinusoidal wave. By taking the measurements at different frequencies, one can get the frequency response of the system (the transfer function). The equivalent circuit scheme for this case is the same as the one shown in Fig. 10. The ac source is labeled as VAC. The results of the simulations using the proposed equivalent circuit model are shown in Fig. 12. As one can see, the results of the small-signal (ac) simulation using the simplified model are in good agreement with those of the 42-elements model as well as with the experimental results.

\section{CONCLUSION}

In this paper, the equivalent circuits are used to describe TEM systems in which direct energy conversion occurs. All nonelectrical processes are emulated by electrical analogies, and their interconnections are represented by dependent sources. The model in Fig. 3 is a tri-port electrical system where two of the ports are an equivalent circuit of the thermal part. Consequently, the model can be implemented as a block in any electrical circuit diagram and run by any electronic circuit simulator.

This paper shows how the parameters of the proposed model can be extracted from manufacturer's data for TEC as well as for TEG products.

The model could be helpful in analyzing the drive requirements of TECs and loading effects of TEGs. Another important application of the proposed model is when the performance of the TEM needs to be analyzed under specific conditions such as heat leakage, nonideal thermal insulation, etc. Using the model, one can select from a commercial TEC catalogue a specific unit that will suit a given application in terms of cooling capability at minimum electrical power drive or estimate the electrical power that can be obtained from a TEG under the expected operating conditions.

The present model is compatible with PSPICE or any other electronic circuit simulators for DC, AC, and TRAN simulations and will thus be an excellent tool for solving problems of temperature control.

Several examples of successful utilization of the model are presented. The paper is based on data of many different manufacturers that were used to reproduce accurately the performance of commercial TEMs.

An important feature of the model is its ability to generate small-signal transfer functions that can be used to design feedback network in temperature control applications.

\section{REFERENCES}

[1] A. F. Ioffe, Semiconductors Thermoelements and Thermoelectric Cooling. London, U.K.: Infoserch Limited, 1957.

[2] S. L. Soo, Direct Energy Conversion. London, U.K.: Prentice-Hall, 1968.

[3] J. Chavez, J. Ortega, J. Salazar, A. Turo, and J. Garcia, "Spice model of thermoelectric elements including thermal effects," in Proc. Instrum. Meas. Technol. Conf., 2000, pp. 1019-1023.

[4] J. P. Holman, Heat Transfer, 7th ed. London, U.K.: McGraw-Hill, 1992.

[5] R. J. Buist and P. G. Lau, "Thermoelectric power generator design and selection from TE cooling module specifications," in Proc. XVI Int. Conf. Thermoelectrics, Dresden, Germany, 1997, pp. 551-554.

[6] P. E. Gray, The Dynamic Behavior of Thermoelectric Devices. New York: Wiley, 1960.

[7] P. E. Bagnoli, C. Casarosa, M. Ciampi, and E. Dallago, "Thermal resistance analysis by induced transient (TRAIT) method for power electronic devices thermal characterization-Part I: Fundamental theory," IEEE Trans. Power Electron., vol. 13, no. 6, pp. 1208-1219, Nov. 1998.

[8] P. G. Lau and R. J. Buist, "Temperature and time dependent finite element model of a thermoelectric couple," in Proc. 15th Int. Conf. Thermoelectrics, 1996, pp. 227-233.

[9] E. De Baetselier, W. Goedertier, and G. De Mey, "A survey of the thermal stability of an active heat sink," Microelectron. Reliab., vol. 37, no. 12, pp. 1805-1812, Dec. 1997.

[10] B. J. Huang and C. L. Duang, "System dynamic model and temperature control of a thermoelectric cooler," Int. J. Refrig., vol. 23, no. 3, pp. $197-207,2000$.

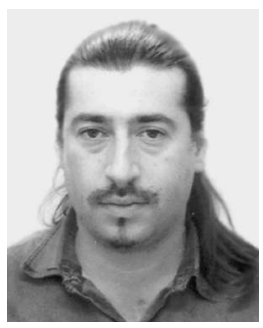

Simon Lineykin received the B.Sc. degree in mechanical engineering and the M.S. degree in electrical engineering from Ben-Gurion University of the Negev, Beer-Sheva, Israel, where he is currently working toward the Ph.D. degree in electrical engineering.

His research interests are modeling and emulation of physical processes and active cooling systems using Peltier effect.

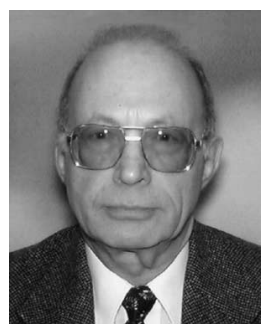

Shmuel (Sam) Ben-Yaakov (M'87) received the B.Sc. degree in electrical engineering from the Technion-Israel Institute of Technology, Haifa, Israel, in 1961, and the M.S. and Ph.D. degrees in engineering from the University of California, Los Angeles (UCLA), in 1967 and 1970, respectively.

$\mathrm{He}$ is presently a Professor with the Department of Electrical and Computer Engineering, Ben-Gurion University of the Negev, Beer-Sheva, Israel, and heads the Power Electronics Group there. His current research interests include power electronics, circuits and systems, electronic instrumentation, and engineering education. He also serves as a consultant to commercial companies in the areas of analog and power electronics. 